\title{
Computed Tomographic Evaluation of Nasopharyngeal Structures of Brachycephalic and Normocephalic Dogs
}

\author{
B. Prakash Kumar ${ }^{1 *}$, Mala Shammi ${ }^{2}$, C Ramani $^{3}$, \\ Geetha Ramesh ${ }^{2}$ and Balagangatharathilagar ${ }^{3}$ \\ ${ }^{1}$ Department of Veterinary Surgery and Radiology, Madras Veterinary College, \\ Chennai -07, India \\ ${ }^{2}$ Director of Distance Education, TANUVAS, Chennai-600035, India \\ ${ }^{3}$ Department of Clinical Medicine, Madras veterinary college, Chennai-07, India \\ *Corresponding author
}

A B S T R A C T

\section{Keywords \\ BAS=brachycephali c airway syndrome $\mathrm{CBC}=$ complete blood count $\mathrm{CT}=$ Computed tomography $\mathrm{HU}=$ Hounsfield unit \\ $\mathrm{I} / \mathrm{V}=$ intravenously $\mathrm{Kvp}=$ kilovoltage peak $\mathrm{mAs}=$ milliampere- second \\ Article Info \\ Accepted: \\ 12 December 2020 \\ Available Online: \\ 10 January 2021}

The present study was carried out to assess the intranasal features and upper air way abnormalities in brachycephalic dogs exhibiting symptoms of brachycephalicair way syndrome (BAS). The study group included the dogs presented with BAS to Madras Veterinary College Teaching Hospital (MVCTH). The dogs were subjected to adetailed clinical examination and diagnostic tests which included complete blood count(CBC), serum biochemical analysis, electrocardiography (ECG), Echocardiography , Radiography prior to Computed tomography (CT). CT was done under general anesthesia with informed consent of the owner. All the dogs were preanesthetized with butorphanol@0.2/kg body weight and diazepam @ 0.25-0.5 / kg body weight. General anesthesia was induced with Propofol @3/kg body weight, intubated and maintained with $2.5 \%$ isoflurane. The dogs were positioned in sternal recumbency with hard palate parallel to the scanning table. CT scan was carried out with slice thickness of $1 \mathrm{~mm}$, the tube rotation time was $0.5 \mathrm{~s}$, with $1 \mathrm{~mm}$ image slice thickness, kvp 100, mAs 150.The images were acquired in bone and soft tissue window(Bone: window width $3500 \mathrm{HU}$, window level $1500 \mathrm{HU}$, soft tissue window width $350 \mathrm{HU}$, and window level 50HU. The CT images showed intranasal abnormalities like deviated nasal septum in all pugs, aberrant turbinate extending into the nasopharyngeal meatus, compared to normocephalic dogs. The soft palate length and thickness were also increased in brachycephalic dog breeds. The hard palate was also longer in brachycephalic dogs compared to the total length of the nose in brachycephalic and normocephalic dog breeds.

\section{Introduction}

Brachycephalic syndrome is a well described combination of hereditary anatomical abnormalities occurring in several breeds of $\operatorname{dogs}$ and cats which include primary abnormalities stenotic nares, elongated soft palate and secondary abnormalities of everted laryngeal saccules and hypoplastic trachea. Improper breed selection has led to extreme brachycephaly resulting in impaired craniofacial development and viscerocranium 
and neurocranium discrepancies. In brachycephalic breeds, specific structural deformities lead to anatomical obstruction of upper airways (Oechering et al., 2016).

Dogs with brachycephalic conformation of the skull are prone to multiple abnormalities of upper air ways like stenotic nares, elongated soft palate, everted laryngeal saccules, hypoplastic trachea. Some animals may show some cartilaginous abnormalities like collapsing trachea and laryngeal collapse. In few instances grossly enlarged tonsils may contribute to upper air way obstruction (Dale 2011). Conventionally, correction of the BAS involves rhinoplasty, staphylectomy, sacculectomy and tonsillectomy when required. In addition to these components of BAS, the presence of nasal turbinates protruding rostrally, rostral aberrant turbinates (RAT) and caudally, caudadl aberrant turbinates (CAT) into the nasopharynx contribute $21 \%$ of upper air way obstruction (Ginn et al., 2008). The presence of aberrant turbinates can be imaged only by CT.Computed tomography is routinely used diagnostic aid for visualizing nasal passages and paranasal sinuses in humans(Hasso 1984). A study in brachycephalic dogs showed severe anatomical changes within the nasal cavity of brachycephalic dogs (Oechering et al., 2007).

The purpose of the study was to study the intranasal components and upper respiratory components in brachycephalic dogs using CT in comparison to normocephalic dogs.

\section{Materials and Methods}

Brachycephalic dogs presented to MVCTH diagnosed with brachycephalic obstructive air way syndrome (BOAS) were taken for the study and compared with normocephalic dogs. All the animals were subjected to CBC, biochemical analysis, blood gas analysis,
ECG, Echocardiography. Informed consent of the owner was obtained before anesthesia for computed tomography (CT).

All the dogs were dogs were cannulated Intravenously and given with butorphanol @ $0.2 \mathrm{mg} / \mathrm{kg}$ body weight I/V (exited dogs were given but orphanol @ $0.2 \mathrm{mg} / \mathrm{kg}$ body weight intramuscularly), followed by diazepam 0.25 $0.5 \mathrm{mg} / \mathrm{kg}$ body weight $\mathrm{I} / \mathrm{V}$. Induction with propofol@ 3mg $/ \mathrm{kg}$ body weight I/V. All the dogs were intubated and connected to boyels anesthetic apparatus maintained with $2 \%$ isofluran. All dogs were monitored carefully during the procedure.

The dogs were positioned in sternal recumbency with hard palate parallel to the scanning table. The tube rotation time was $0.5 \mathrm{~s}$, with $1 \mathrm{~mm}$ image slice thickness, kvp $100, \mathrm{mAs} 150$. The images were acquired in bone and soft tissue window(Bone: window width $3500 \mathrm{HU}$, window level $1500 \mathrm{HU}$, soft tissue window width $350 \mathrm{HU}$, and window level 50HU) (Fig. 1).

\section{Results and Discussion}

Comparison of the CT slices showed clear structural differences between pugs and other normocephalic dog breeds. The nasal passages were clearly demarcated in normocephalic dog breeds but in pugs the nasopharyngeal meatus space is reduced in brachycephalic dog breeds compared to nornocephalic dog breeds. An important finding was aberrant turbinates projecting into the nasopharyngeal meatus, which was completely absent in normocephalic dog breeds. All the brachycephalic dogs showedsevere deviation in nasal septum which is not seen in normocephalic dog breeds. Compared to length of the nasal cavity, the hard palate and soft palate are longer in brachycephalic dogs than in normocephalic dogs. The mean length of soft 
palate in brachycephalic dogs $39.16 \pm 1.52$ and thickness

$9.17 \pm 0.41 \quad$ compared to normocephalic dog length $34.630 \pm 0.816$ and thickness was $7.666 \pm 0.559$.

Table.1 Length and thickness of soft palate in brachycephalic breeds

\begin{tabular}{|c|c|c|}
\hline & $\begin{array}{c}\text { Length } \\
(\mathbf{m m})\end{array}$ & $\begin{array}{c}\text { Thickness } \\
(\mathbf{m m})\end{array}$ \\
\hline $\mathbf{1}$ & 30.60 & 09.10 \\
\hline $\mathbf{2}$ & 39.50 & 10.10 \\
\hline $\mathbf{3}$ & 46.20 & 08.20 \\
\hline $\mathbf{4}$ & 33.90 & 10.40 \\
\hline $\mathbf{5}$ & 41.60 & 12.00 \\
\hline $\mathbf{6}$ & 35.70 & 09.60 \\
\hline $\mathbf{7}$ & 38.70 & 07.40 \\
\hline $\mathbf{8}$ & 45.60 & 07.40 \\
\hline $\mathbf{9}$ & 38.70 & 06.40 \\
\hline $\mathbf{1 0}$ & 40.50 & 10.10 \\
\hline $\mathbf{1 1}$ & 48.0 & 09.40 \\
\hline $\mathbf{1 2}$ & 30.60 & 09.10 \\
\hline $\mathbf{1 3}$ & 39.50 & 10.10 \\
\hline
\end{tabular}

Mean $\quad 39.16 \pm 1.52 \quad 9.17 \pm 0.41$

Table. 2 Length and thickness of soft palate in normocephalic breeds

\begin{tabular}{|c|c|c|}
\hline S No & $\begin{array}{c}\text { Length } \\
\text { (mm) }\end{array}$ & $\begin{array}{c}\text { Thickness } \\
\text { (mm) }\end{array}$ \\
\hline 1 & 36 & 10.1 \\
\hline 2 & 30.2 & 8.7 \\
\hline 3 & 37.5 & 3.3 \\
\hline 4 & 33.6 & 8.3 \\
\hline 5 & 32.4 & 6.1 \\
\hline 6 & 36.4 & 9.6 \\
\hline 7 & 34.2 & 7.8 \\
\hline 8 & 36.2 & 8.2 \\
\hline 9 & 30.0 & 10.7 \\
\hline 10 & 31.3 & 8.2 \\
\hline 11 & 35.8 & 6.5 \\
\hline 12 & 37.4 & 5.92 \\
\hline 13 & 39.2 & 6.24 \\
\hline
\end{tabular}


Fig.1

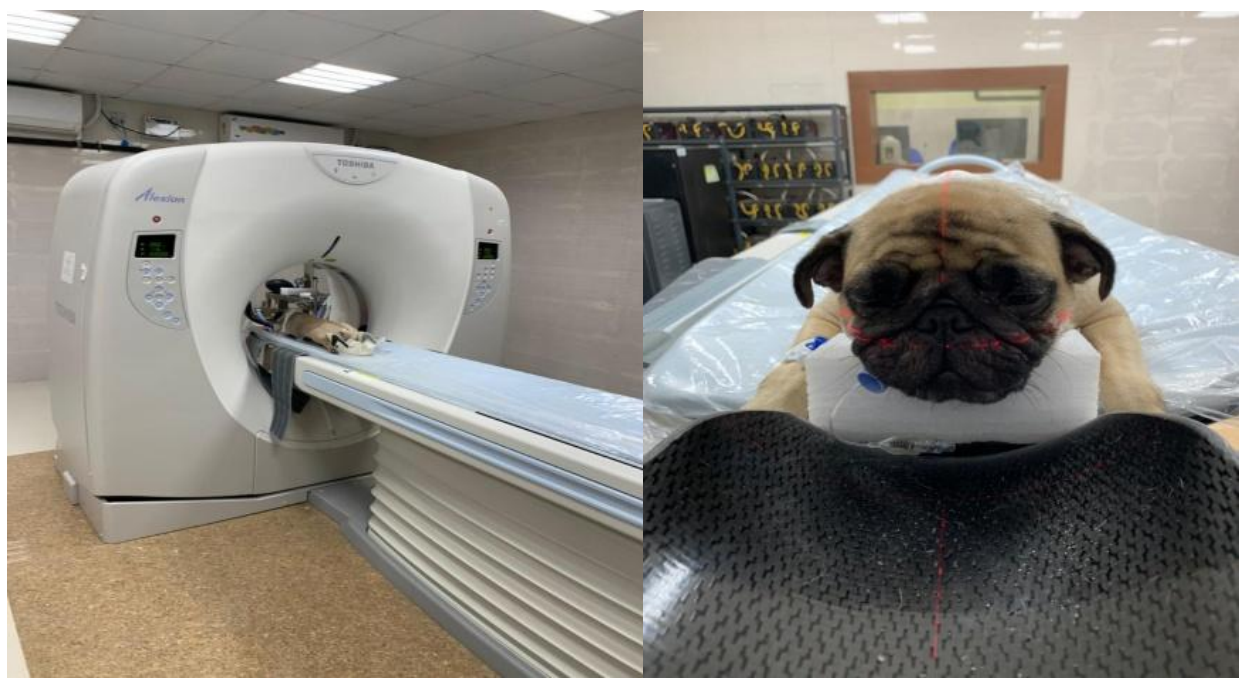

CT image of saggital section of head Soft palate in brachycephalic dog showing elongated

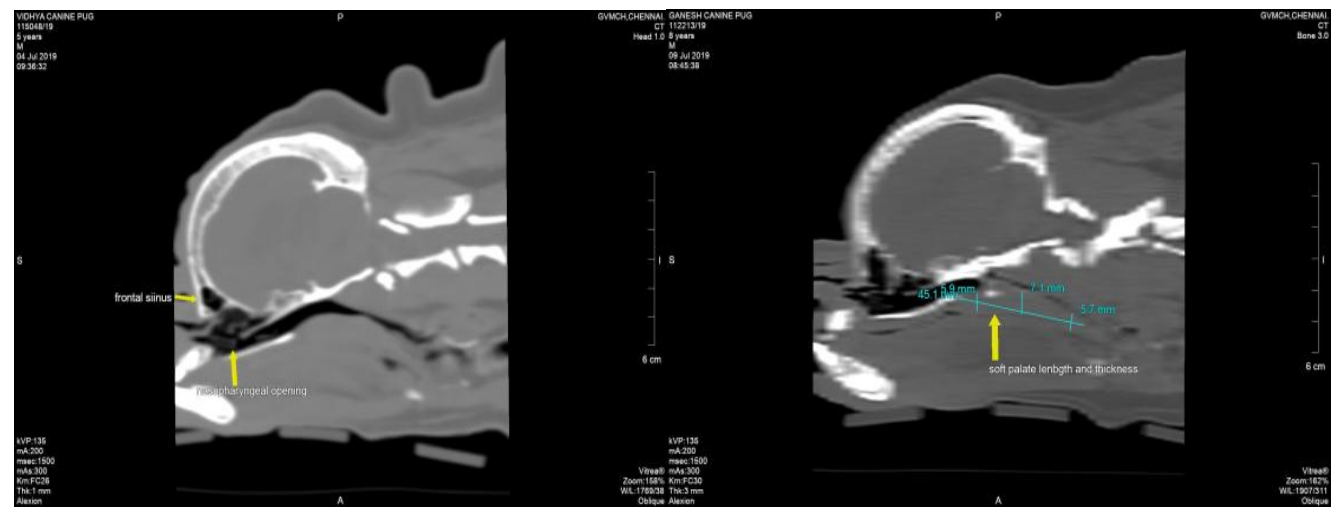

Aberrant turbinates were found in nasopharyngeal meatus

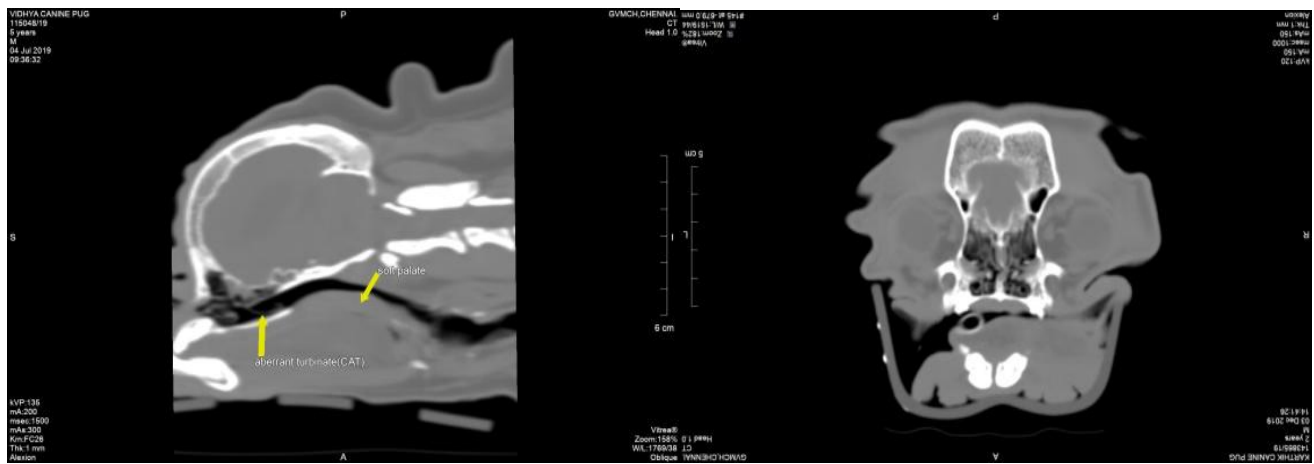


Deviated nasal septum is found in almost all cases

Brachycephalic dog

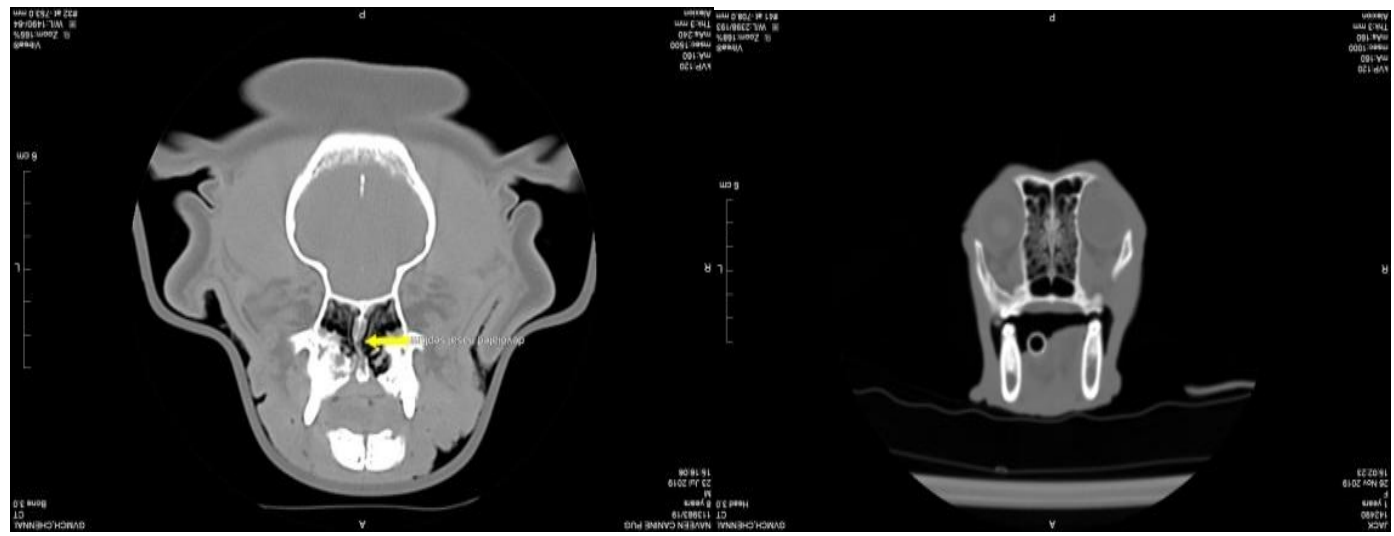

Aberrant turbinates

Brachycephalic dog

normocephalic dog

normocephalic dog

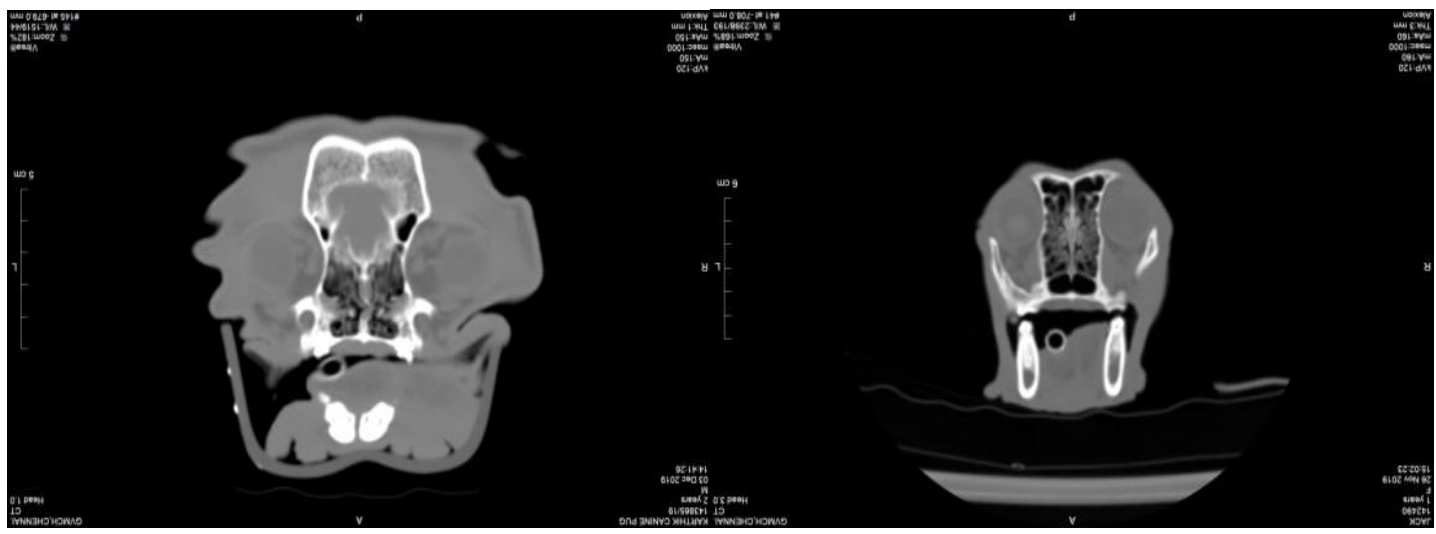

All the 13 brachycephalic dogs showed increased length and thickness of the soft palate compared to normocephalic dogs which narrows the nasopharyngeal airway causing severe respiratory distress. Earlier, increase in the length of soft palate was considered to be the main contributory factor for obstructing the air way, however thickness of soft palate was also found to further reduce the nasopharyngeal meatus aggravating respiratory distress. Findji and Dupre 2008 reported increased thickness of Soft palte in dogs with BOAS. Grand and Beuro in a study in 2011 suggested that a thickened soft palate was a new component in BOAS. The increase in thickness was attributed to hypertrophied palatal muscles, mucosal edema or both. These changes could be a response to increased intranasal airflow resistance at the level of nares, vestibulum and cavum nasi (Oechering et al., 2007).

Extremely shortened nose and the minimal space for turbinate growth could be contributory reasons for pugs showing the highest prevalence of aberrant turbinate growth. Similar findings were reported by Ginn et al., 2008.

An important finding in brachycephalic dogs was the protrusion of the conchae into intranasal space both caudally and rostrally. 
The presence of aberrant cranial and rostral turbinates was a typical finding $\mathrm{I} n$ brachycephalic dogs (Oechering et al., 2016).

Deviation of the nasal septum was seen all cases which coincided with the findings of Oechering et al., 2016.They also reported that this abnormality was seen in only $30 \%$ of normacephalic dogs. It is therefore appears that deviated nasal septum in common in brachycephalic dogs especially pugs. It is known that deviated nasal septum results in nasal obstruction caused by increased air resistant to the convex side and hypertrophy of concha in concave side in humans Grutznmacher et al., (2006).

Brachycephalic dog breeds with brachycephalic syndrome have morphological intranasal abnormalities such as aberrant turbinates, deviated nasal septum, reduced nasopharyngeal meatus elongated and thickened softpale. CT scan is an important and specific diagnostic aid to evaluate upper airway in brachycephalic dogs under general anesthesia.

\section{Acknowledgements}

Authors are thankful to Department of veterinary surgery and radiology, madras veterinary college, Chennai-07 for constant support during the entire research.

\section{References}

Findji, L. and Dupre, G., 2008. Folded flap palatoplasty for treatment of elongated soft palates in 55 dogs. Wiener
Tierarztliche Monatsschrift, 95(3/4), p.56.

Ginn, J.A., Kumar, M.S.A., McKiernan, B.C. and Powers, B.E., 2008. Nasopharyngeal turbinates in brachycephalic dogs and cats. Journal of the American Animal Hospital Association, 44(5), pp.243-249.

Grützenmacher, S., Robinson, D.M., Gräfe, K., Lang, C. and Mlynski, G., 2006. First findings concerning airflow in noses with septal deviation and compensatory turbinate hypertrophy-a model study. ORL, 68(4), pp.199-205.

Hasso, A.N., 1984. CT of tumors and tumorlike conditions of the paranasal sinuses. Radiologic Clinics of North America, 22(1), p.119.

Oechtering, G.U., Pohl, S., Schlueter, C., Lippert, J.P., Alef, M., Kiefer, I., Ludewig, E. and Schuenemann, R., 2016. A novel approach to brachycephalic syndrome. 1. Evaluation of anatomical intranasal airway obstruction. Veterinary Surgery, 45(2), pp.165-172.

Oechtering, T.H., Oechtering, G.U. and Nöller, C., 2007. Structural characteristics of the nose in brachycephalic dog breeds analysed by computed tomography. Tierärztliche Praxis Ausgabe K: Kleintierel Heimtiere, 35(03), pp.177-187.

Rasteiro, A.M.F., 2015. In vitro comparative studies on new targets for cancer therapy, and characterization of their biomolecular effects (Doctoral dissertation, Universidade de Lisboa. Faculdade de Medicina Veterinária).

\section{How to cite this article:}

Prakash Kumar, B., Mala Shammi, C Ramani, Geetha Ramesh and Balagangatharathilagar. 2021. Computed Tomographic Evaluation of Nasopharyngeal Structures of Brachycephalic and Normocephalic Dogs. Int.J.Curr.Microbiol.App.Sci. 10(01): 1151-1157. doi: https://doi.org/10.20546/ijcmas.2021.1001.139 